\title{
ULTRASTRUCTURAL FINDINGS IN SOLAR RETINOPATHY
}

\author{
M. W. HOPE-ROSS, G. J. MAHON, T. A. GARDINER and D. B. ARCHER \\ Belfast, Northern Ireland
}

\begin{abstract}
SUMMARY
This study documents the ultrastructural findings in a case of solar retinopathy, 6 days after sungazing. A malignant melanoma of the choroid was diagnosed in a 65 year-old man. On fundoscopy, the macula was normal. The patient agreed to stare at the sun prior to enucleation. A typical solar retinopathy developed, characterised by a small, reddish, sharply circumscribed depression in the foveal area. Structural examination of the fovea and parafovea revealed a spectrum of cone and rod outer segment changes including vesiculation and fragmentation of the photoreceptor lamellae and the presence of discrete 100-120 nm whorls within the disc membranes. Many photoreceptor cells, particularly the parafoveal rods, also demonstrated mitochondrial swelling and nuclear pyknosis. Scattered retinal pigment epithelial cells in the fovea and parafovea showed a degeneration characterised by loss of plasma membrane specialisations, swelling of the smooth endoplasmic reticulum and changes in the fine structure of the lipofuscin granules. The good visual prognosis in solar retinopathy was attributed to the resistance of the foveal cone cells to photochemical damage.
\end{abstract}

Eclipse watching is the major cause of solar retinopathy. ${ }^{1}$ It has also been described in sunbathers, pilots, military recruits, in patients with mental illness and following religious rituals. ${ }^{2}$ Following exposure, patients complain of decreased visual acuity and central scotomas.

Fundoscopy in the early stages reveals a small yellow spot in the centre of the fovea. This fades and is replaced by areddish spot surrounded by a pigment halo. After approximately 1-2 weeks a small, red, sharply circumscribed lamellar hole develops in the foveal region. This lesion is frequently permanent, and is characteristic of solar retinopathy. Fluorescein angiography is typically normal, but in the acute stages a small focal area of staining may be present.

From: Royal Victoria Hospital, Grosvenor Road, Belfast BT12 6BA, Northern Ireland.

Correspondence to: Miss M. W. Hope-Ross, Birmingham and Midland Eye Hospital, Church Street, Birmingham B3 2NS, UK.
The prognosis for recovery in solar retinopathy is good and the majority of patients regain pre-exposure visual acuity. ${ }^{3}$ However, central scotomas frequently persist.

The purpose of this paper is to document the ultrastructural findings in a case of solar retinopathy.

\section{CASE REPORT AND METHODS}

A 65-year-old man presented to the Ophthalmic Department of the Royal Victoria Hospital, Belfast, complaining of a 10-week history of floaters affecting the right eye. Examination revealed best corrected visual acuity was right eye 6/9 and left eye 6/4. Fundoscopy of the right eye revealed an inferonasal peripapillary choroidal malignant melanoma, measuring $9 \mathrm{~mm}$ in diameter. A fluorescein angiogram confirmed the presence of a choroidal malignant melanoma; the macula was normal (Fig. 1). Systemic investigations did not reveal any evidence of metastatic spread.

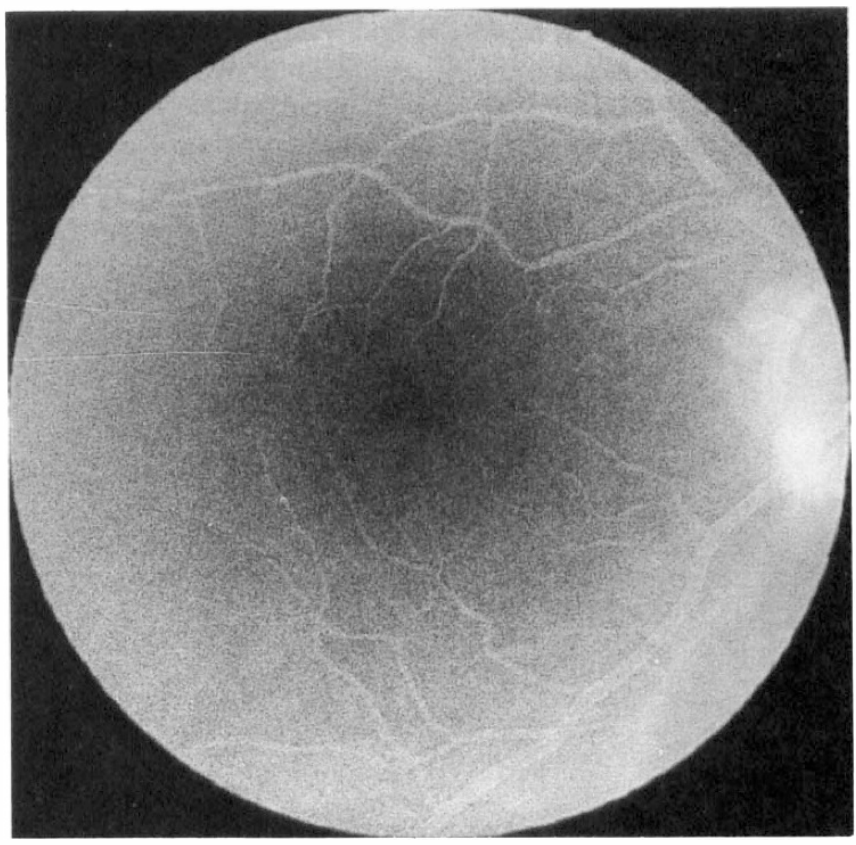

Fig. 1. Fluorescein angiogram of the right eye prior to sungazing shows no abnormality of the fovea. 


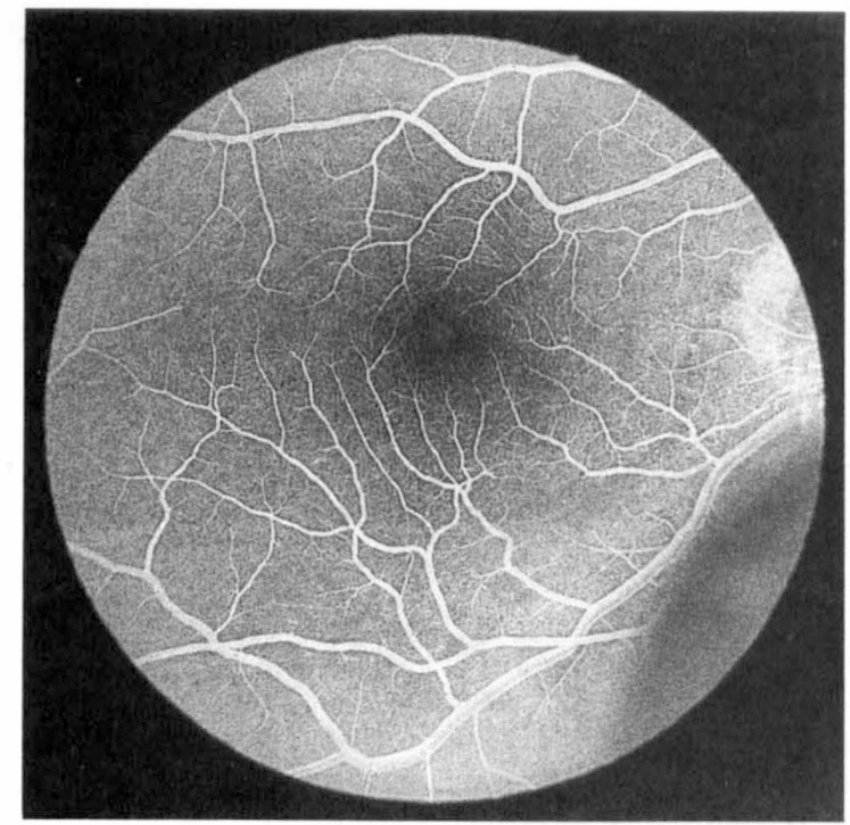

Fig. 2. Fundoscopy of the right eye following sungazing show's a small, reddish, sharply circumscribed depression in the foveal area.

In view of the size of the tumour and the proximity to the optic disc, it was decided to enucleate the right eye. Prior to enucleation, the patient agreed to stare at the sun. The pupil was dilated with guttae cyclopentolate $1 \%$ and measured $5 \mathrm{~mm}$. The left eye was occluded and he stared at the sun uninterruptedly for 10 minutes. He described that immediately afterwards he could see a black spot in front of the right eye and visual acuity in this eye was $6 / 24$. Six days later visual acuity in the right eye was $6 / 9$; no scotomas were detected on Amsler grid testing. Fundoscopy revealed the typical appearance of solar retinopathy (Fig. 2). A repeat fluorescein angiogram of the macula showed no obvious abnormality (Fig. 3). The right eye was enucleated, following which small tissue samples of retina and attached choroid were dissected from the macula and from the cen-

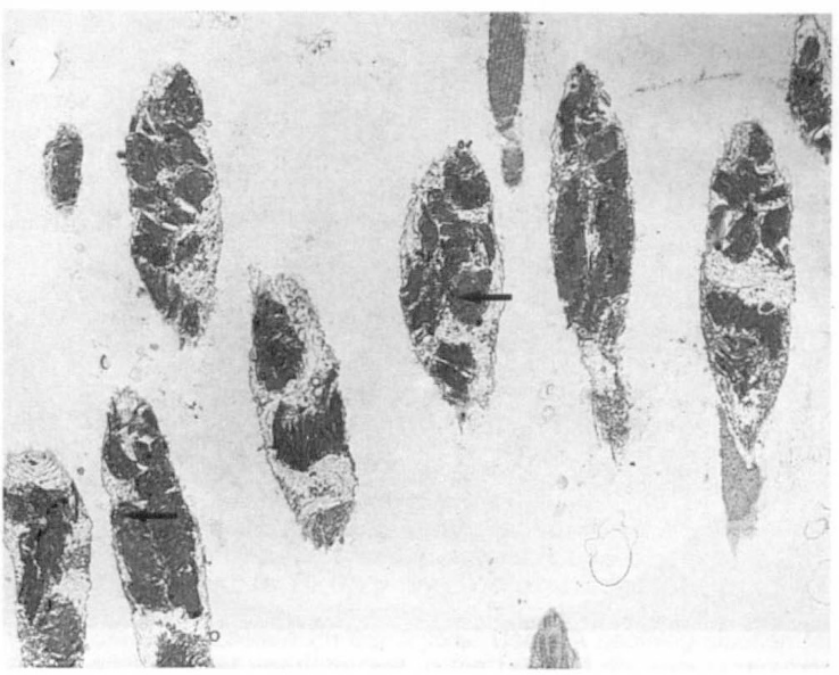

Fig. 4 (a). Cone outer segments in the fovea show swelling, fragmentation, vesiculation and many $100-120 \mathrm{~nm}$ membranous whorls (arrows). $\times 5000$.

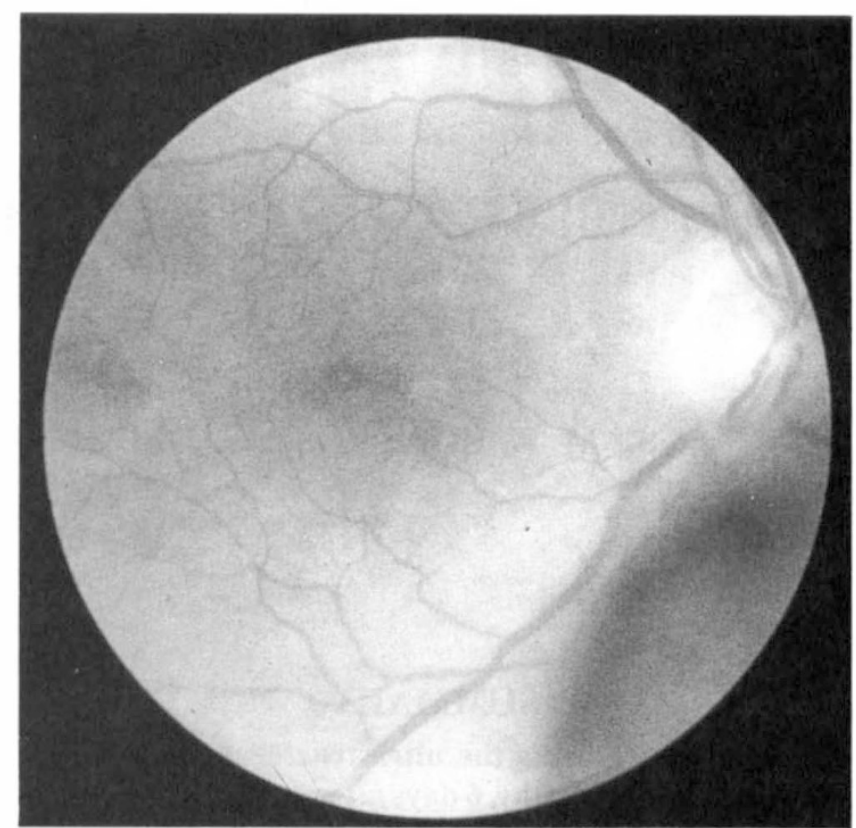

Fig. 3. Fluorescein angiogram following solar exposure fails to show' any abnormality of the macula.

tral and peripheral retina uninvolved by the tumour. The tissue samples were fixed overnight in $2.5 \%$ glutaraldehyde in $0.1 \mathrm{~mol} / \mathrm{l}$ cacodylate buffer, $\mathrm{pH} 7.2$. The tissue blocks were post-fixed in $2 \%$ osmium tetroxide, dehydrated in ascending grades of alcohol and embedded in epoxy resin for electron microscopy sectioning. The remainder of the eye, including the tumour, was fixed in $10 \%$ formalin for histological examination.

\section{RESULTS}

In the foveal and parafoveal regions the photoreceptor cells showed swelling of their outer segments with fragmentation and vesiculation of the photoreceptor lamellae (Fig. 4). Large numbers of discrete 100-200 $\mathrm{nm}$ whorls were present within the disc membranes (Fig. 5). It was notable that in some outer segments the whorls were the only obvious fine

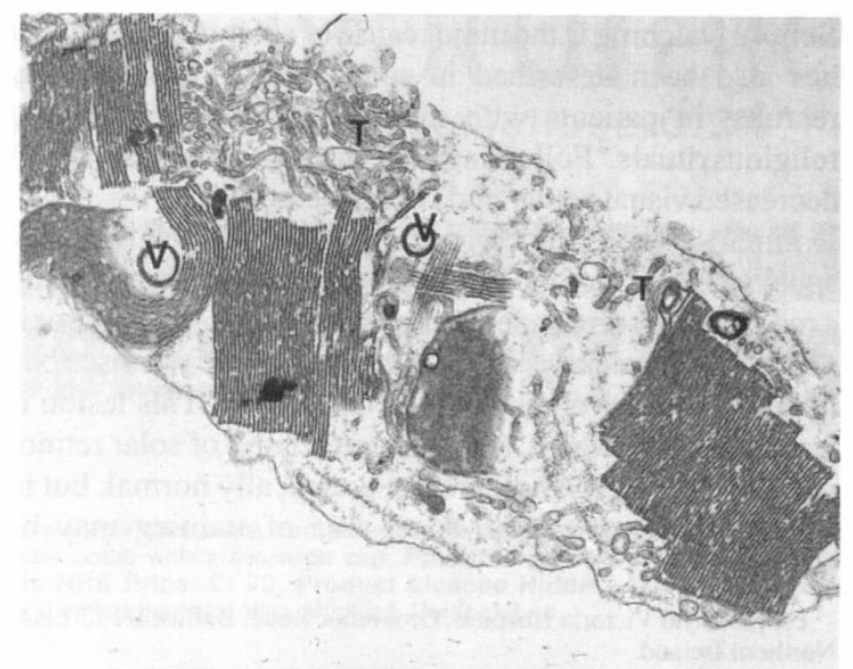

Fig. 4 (b). Cone outer segment at higher magnification shows fragmentation of the disc membranes with vesicular $(V)$ and tubular $(T)$ profiles. $\times 24450$. 


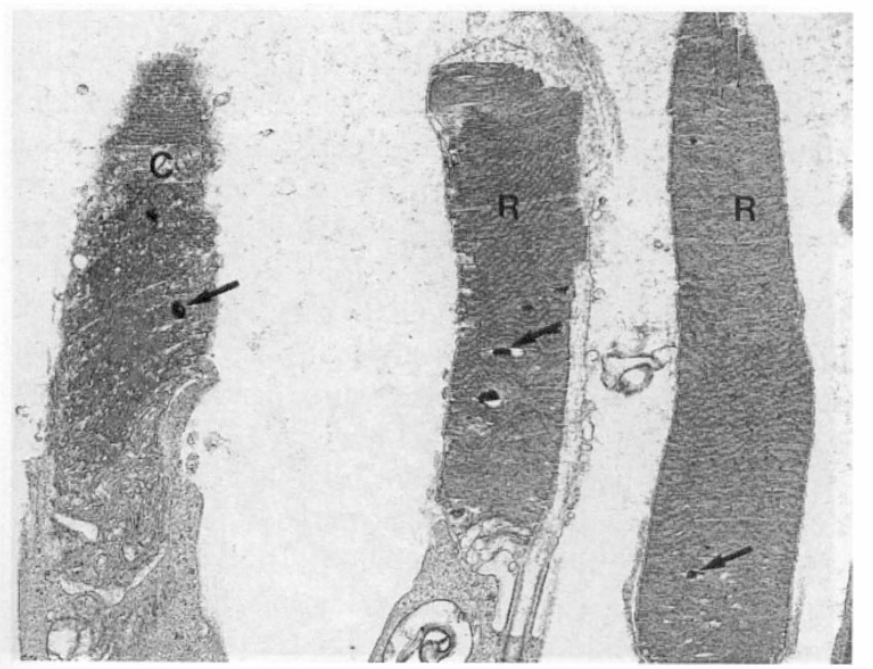

Fig. 5. Cone $(C)$ and rod $(R)$ outer segments in the parafovea show 100-120 nm membranous whorls (arrows). $\times 15000$.

structural abnormality. Some outer segments also showed gross kinking and folding, but as the neural retina had undergone artefactual detachment during processing these changes were attributed to simple mechanical disruption.

Swollen mitochondria were present in the inner segments of a large number of the photoreceptors in the fovea and parafovea, especially in the rod cells of the parafovea (Fig. 6a), but were not encountered in the remainder of the central retina (Fig. 6b).

A small number of the foveal and parafoveal cones showed increased granularity of their nuclear chromatin (Fig. 7). In contrast, a large proportion of the rod cells in the parafovea demonstrated frank pyknosis (Fig. 8).

Most of the retinal pigment epithelial (RPE) cells in the macula appeared normal. The RPE cell cytoplasm had a high content of lipofuscin and melanofuscin, a change consistent with the patient's age. Approximately 5\% of the RPE cells in the fovea and parafovea demonstrated a spectrum of degenerative changes which included loss of the apical microvilli and basal plasmalemmal infoldings, swelling of the smooth endoplasmic reticulum and Golgi

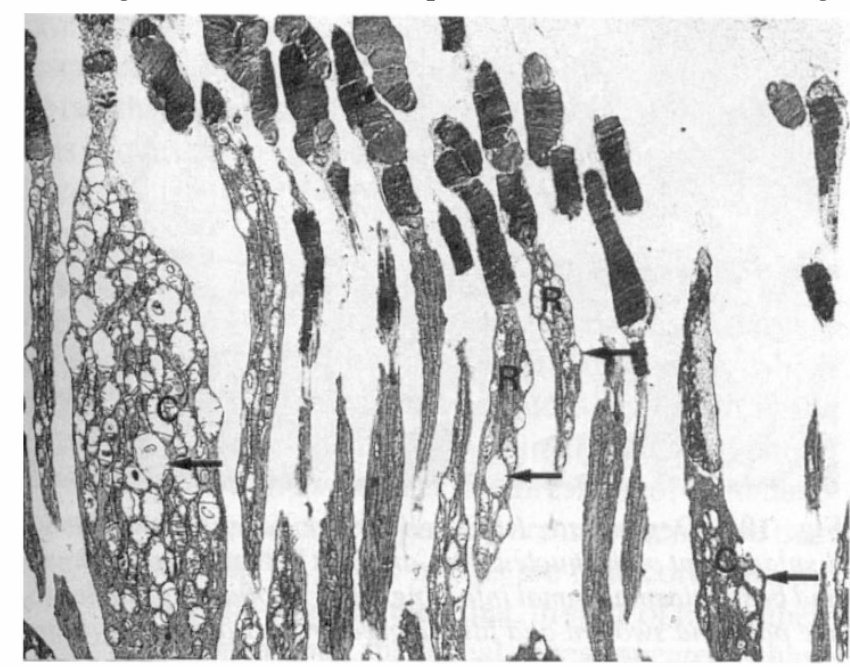

Fig. 6 (a). Inner segments of rods $(R)$ and cones $(C)$ in the parafovea show swollen mitochondria (arrows). $\times 4000$. elements, and either apical or lateral displacement of the cell nucleus. Also, the lipofuscin and melanofuscin granules in such cells were unusually electron-lucent in comparison with those in neighbouring normal RPE cells (Fig. 9). This phenomenon was particularly evident in the melanofuscin complexes, where the enclosed melanin granules, which were consistently electron-opaque, could easily be identified within the 'pale' matrix of the bodies. The 'pale' lipofuscin granules appeared swollen and filled the bulk of the cytoplasm in the affected cells (Fig. 10).

The inner retinal layers in the macula and throughout the rest of the retina showed no obvious fine structural abnormalities. Bruch's membrane and the choriocapillaris were unremarkable in all regions examined by electron microscopy.

\section{DISCUSSION}

This study demonstrates the ultrastructural findings in a case of solar retinopathy, 6 days after a solar injury. The literature contains two previous histopathological studies of the human eye following sun gazing. ${ }^{4.5}$ An ultrastructural study by Tso et al. found, in agreement with the current report, that pathological changes were largely confined to the outer retina. They described alterations in the photoreceptor outer segments which, although not illustrated, appear similar to the findings in the present study and in addition noted accumulations of dense bodies in the inner segments. In contrast to the present study they also found depigmentation of the RPE and pathological changes involving Bruch's membrane and the choriocapillaris. A light microscopical study of an eye enucleated 6 months after a solar insult was reported by Gass, who described photoreceptor cell loss and depigmentation of the RPE.

It was formerly thought that solar retinopathy resulted from a thermal injury, and although it is now appreciated that the maximum temperature increase at the level of the retina $\left(4^{\circ} \mathrm{C}\right)$ is insufficient to cause coagulative necrosis, ${ }^{6}$ the effect of temperature should not be dismissed completely as increases of this order have been shown to initiate the expression of heat shock proteins in retinal cells. ${ }^{7}$ How-

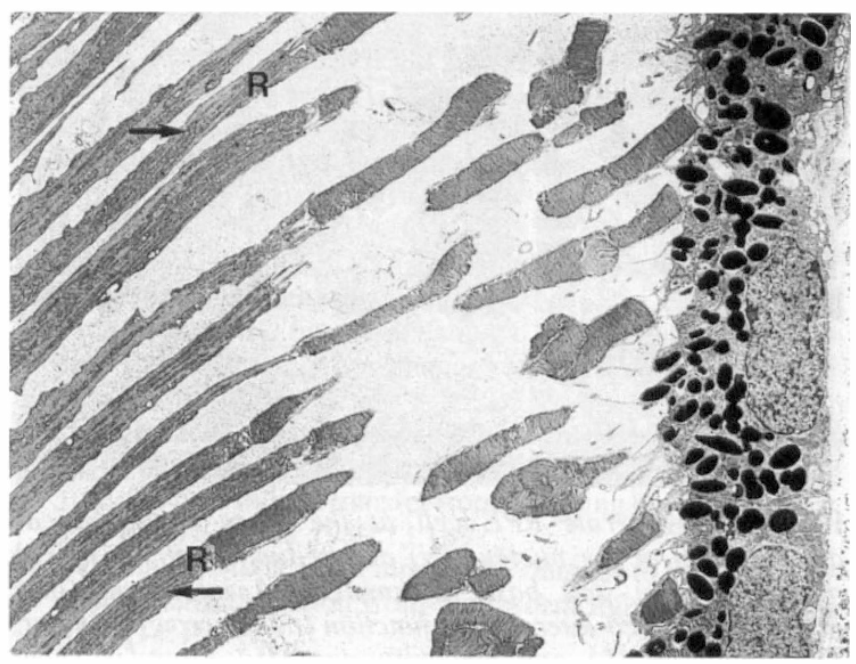

Fig. 6 (b). The mitochondria (arrow's) in rods $(R)$ from the peripheral retina appear normal. $\times 3500$. 


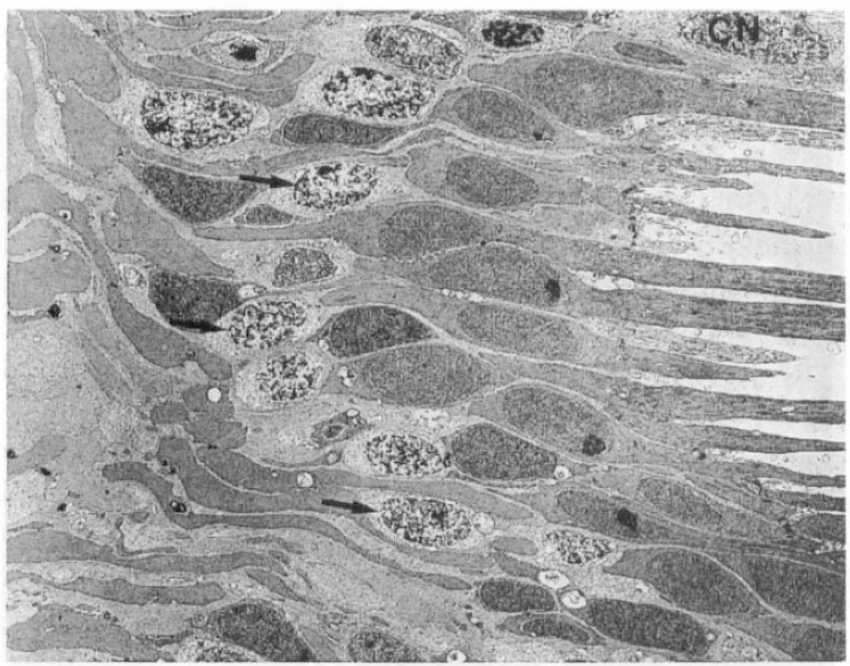

Fig. 7. On the parafovea pyknotic rod nuclei (arrows) are present in the inner arcade of the outer nuclear layer. A cone nucleus $(C N)$ shows increased granularity of the chromatin. $\times 1500$.

ever the weight of current evidence suggests that the main causative factor in solar retinopathy is a photochemical injury mediated by highly reactive free radicals. ${ }^{8}$

In the present study, retinal injury was most severe in the photoreceptor cells of the fovea and the parafovea, especially in the rod cells of the parafovea where the widespread occurrence of pyknotic nuclei heralded the impending death of the affected cells. The photoreceptor outer segment membranes appeared more susceptible to solar radiation damage than any other cell component; rods and cones were similarly affected. Almost all of the outer segment aberrations described above may be produced artefactually through inappropriate fixation, physical trauma and chemical or thermal insult. However, as the changes were confined to the fovea and parafovea and coincided with

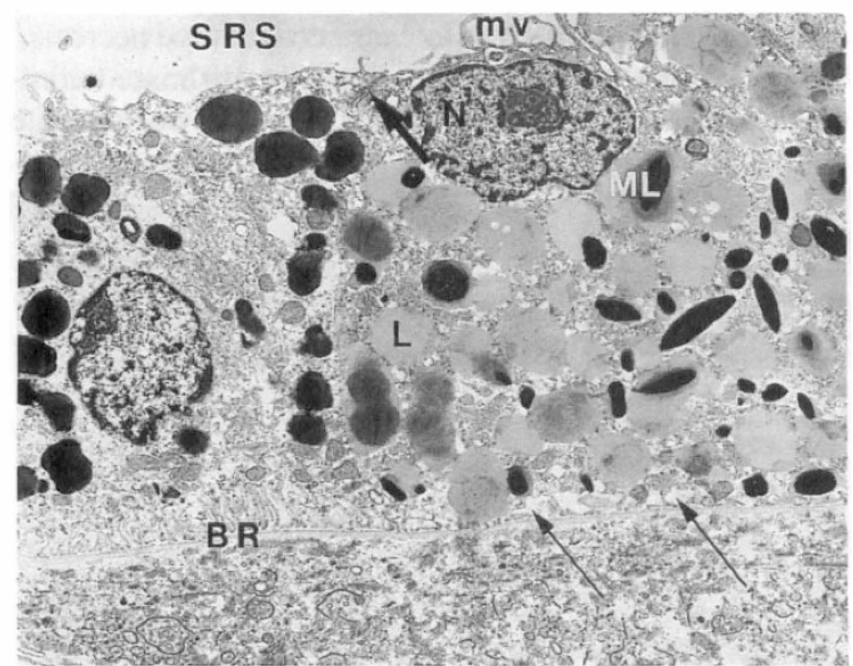

Fig. 9. Degenerate RPE cell at the fovea shows apical displacement of the nucleus $(N)$ and reduction of the apical microvilli $(\mathrm{mv})$ and basal plasmalemmal infoldings (thin arrows). An apico-lateral tight junction (thick arrow) is intact. The lipofuscin (L) and melanofuscin $(M L)$ granules show reduced osmiophilia in comparison with those of an adjacent cell. BR, Bruch's membrane; SRS, subretinal space. $\times 6500$.

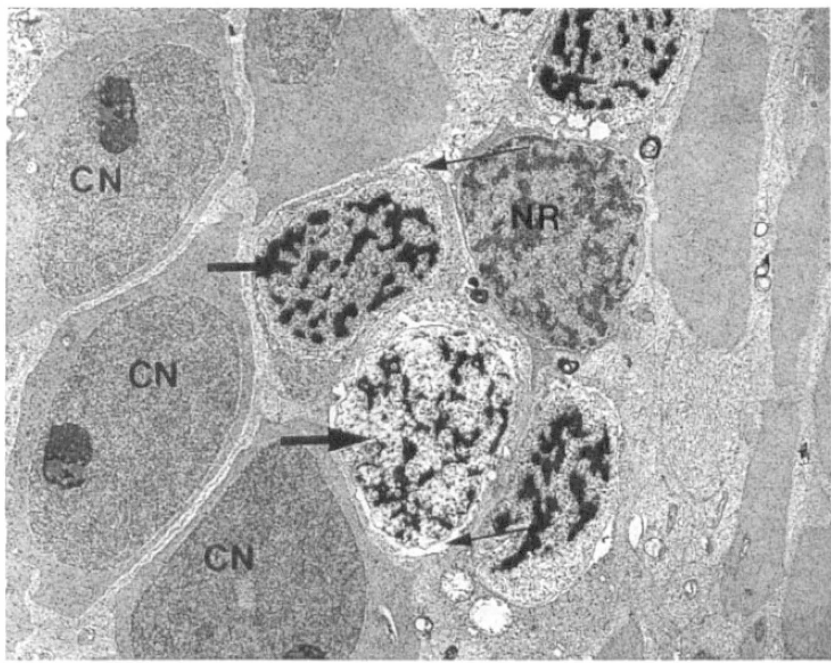

Fig. 8. In the parafovea pyknotic rod nuclei (thick arrows) show coarsely granular chromatin and swelling of the nuclear envelope (thin arrows). A normal rod nucleus (NR) and several normal cone nuclei $(C N)$ are also present. $\times 4500$.

pyknotic rod nuclei in the parafovea, they were attributed to solar injury. The susceptibility of the photoreceptor membranes to intense light is now thought to be due to their high content of polyunsaturated and di-polysaturated fatty acids. ${ }^{9}$ These lipids are particularly vulnerable to peroxidation by oxygen-derived free radicals which are generated in living cells by various forms of electromagnetic radiation including visible light. ${ }^{10.11}$ Under normal circumstances endogenous scavenging systems protect the retina from free radical attack. ${ }^{12.13}$ However, overexposure to any appropriate radiation may exhaust the protective mechanisms and lead to cell injury.

Almost all the ultrastructural sequelae of solar radiation damage to the photoreceptor outer segments noted in the present study have been documented in previous investiga-

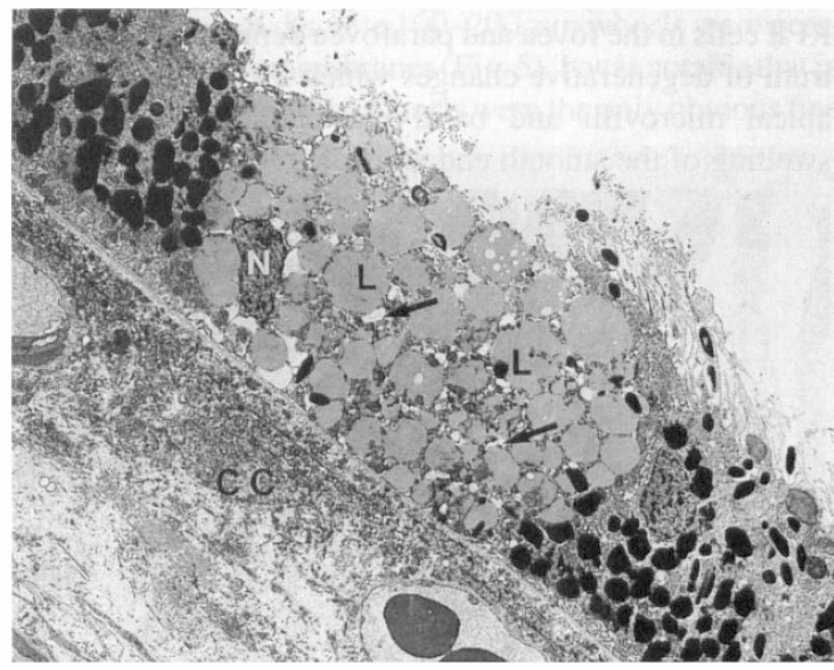

Fig. 10. Degenerate RPE cell at the fovea shows lateral displacement of the nucleus $(N)$, and loss of the apical microvilli and basal plasmalemmal infoldings. The lipofuscin granules $(L)$ are pale and swollen and fill the greater part of the cytoplasm. Clear regions within the cytoplasm represent swollen cisternae of the smooth endoplasmic reticulum (arrows). The neural retina is artefactually detached. CC, choriocapillaris. $\times 2500$. 
tions of light damage to the retina. ${ }^{14}$ However, the occurrence of discrete $100-120 \mathrm{~nm}$ whorls within the disc membranes is of interest as nearly identical structures have been observed in the photoreceptor outer segments of rats exposed to X-radiation. ${ }^{15}$ This is not surprising as cell damage from both visible light and X-rays is mediated by free radicals.

The mitochondrial swelling in the photoreceptor cells of the fovea and parafovea was attributed to solar radiation damage as the change was not encountered elsewhere in the retina. Mitochondria may be vulnerable to light damage through their content of metals such as iron and copper, which under appropriate conditions catalyse the generation of free radicals. ${ }^{16}$ It is possible that the numerous large mitochondria in the photoreceptors, when over-irradiated with visible light, may act as sources of free radicals which damage not only the mitochondria but the cells which contain them.

The nuclear pyknosis which was so evident in rod cells of the parafovea may have resulted from free radical damage to the nuclear chromatin at the time of exposure to the sun. However, nuclear pyknosis is a terminal morphological sign shared by most if not all cells during cell death. Therefore it is also possible that the rods were undergoing death due to overwhelming destruction of key cytoplasmic components such as mitochondria. Although the architecture of the photoreceptor cells precluded any direct correlation of the pyknotic nuclei in the outer nuclear layer with inner segments showing swollen mitochondria, because these changes were confined to the fovea and parafovea and occurred in a similar proportion of the cells, it is possible that both features were represented within the same population.

The fine structural anomalies noted in some RPE cells of the fovea and parafovea suggested that these cells may eventually have died and detached from Bruch's membrane. However, as only a small proportion of the RPE appeared to have suffered, it is probable that such microscopic defects could be easily repaired by cell sliding and/or local division. Alternatively, the affected cells may have survived but lost their pigment as in the cases described by Tso and Gass. This possibility is supported by the fact that the lipofuscin and melanofuscin granules of the cells in question were clearly undergoing some qualitative change which may have resulted in their extrusion from the cells.

The present study has shown that the favourable prognosis for visual recovery in solar retinopathy is largely attributable to the resilience of the central cones, which were seen to survive in spite of obvious cell death in the adjacent rods. Several studies on animal models of photic retinopathy have shown that at the threshold of light damage to the retina, the cone segments are more sensitive than those of the rods. ${ }^{17,18}$ These results are in accord with the observation in the present study that, in spite of their otherwisehealthy appearance, the foveal cones appeared to have incurred at least as much outer segment damage as the parafoveal rods. However, none of the experimental studies compared photoreceptor cell survival, and as the outer segment membranes are continuously renewable, the visual prognosis ultimately depends on cell survival. Why such similar cells with a common embryonic origin and shared environment should exhibit such a differential sensitivity to light damage is difficult to explain. However, a comparison of their antioxidant protection systems and the free radical generating or scavenging properties of the photopigments found in each cell type may help clarify the observed differences in their vulnerability to photochemical insult.

Key words: Photoreceptors, Phototoxicity, Retinal pigment epithelium, Solar retinopathy, Transmission electron microscopy.

\section{REFERENCES}

1. Penner R. Eclipse blindness. Am J Ophthalmol 1966;61: 1452-7.

2. Hope-Ross M, Travers T, Mooney D. Solar retinopathy following religious rituals. Br J Ophthalmol 1988;12:931-4.

3. MacFaul PA. Visual prognosis after solar retinopathy. Br J Ophthalmol 1969;53:534-41.

4. Tso MOM, LaPiana FG. The human fovea after sun gazing. Trans Am Acad Ophthalmol Otolaryngol 1975;79:788-95.

5. Gass JDM. Stereoscopic atlas of macular diseases: diagnosis and treatment. St Louis: Mosby, 1977:322-5.

6. White TJ, Mainster MA, Wilson PW. Chorioretinal temperature increases from solar observation. Bull Math Biophys 1971;33:1-17.

7. Wakakura M, Foulds WS. Response of cultured Müller cells to heat shock: an immunocytochemical study of heat shock and intermediate filament proteins in response to temperature elevation. Exp Eye Res 1989;48:337-50.

8. Woodford BJ, Tso MOM, Lam K.-W. Reduced and oxidised ascorbate in guinea pig retina, under normal and light exposed conditions. Invest Ophthalmol Vis Sci 1983;24: 862-7.

9. Anderson RE, Andrews LD. Biochemistry of retinal photoreceptor membranes in vertebrates and invertebrates. In: Westfall JA, editor. Visual cells in evolution. York: Raven Press, 1982.

10. Kagan VE, Shvedova AA, Novikov KN, Kozlov YP. Light induced free radical oxidation of membrane lipids in photoreceptors of frog retina. Biochim Biophys Acta 1973;330:76

11. Wiegand RD, Giusto NM, Anderson RE. Lipid changes in albino rat rod outer segments following constant illumination. In: Clayton R, Heywood I, Reading H, Wright A, editors. Biology of normal and genetically abnormal retinas. New York: Academic Press, 1982:121-8.

12. Organisciak DT, Wang HM, Kon AL. Ascorbate and glutathione levels in the developing normal and dystrophic rat retina: effect of intense light exposure. Curr Eye Res 1984;3:257.

13. Mittag T. Role of oxygen radicals in ocular inflammation and cellular damage. Exp Eye Res 1984;39:759.

14. Tso MOM, Woodford BJ. Effect of photic injury of the retinal tissues. Ophthalmology 1983;90:952-63.

15. Amoaku WMK, Frew L, Mahon GJ, Gardiner TA, Archer DB. Early ultrastructural changes after low dose X-radiation in the retina of the rat. Eye 1989;3:638-46.

16. Schaich KM. Free radical initiation in proteins and amino acids by ionising and ultraviolet radiations and lipid oxidation. III. Free radical transfer from oxidising lipids. CRC Crit Rev Food Sci Nutr 1980;13:189.

17. Marshall J, Mellario J, Palmer DA. Damage to the pigeon by moderate illumination from fluorescent light. Exp Eye Res 1972;14:164-9.

18. Sykes SM, Robinson G, Woxler M, Kuwabara T. Damage to the monkey retina by broad spectrum fluorescent light. Invest Ophthalmol Vis Sci 1981;20:425-33. 